\title{
The Turán-Kubilius inequality for integers free of large prime factors (II)
}

by

\author{
Ti Zuo XUAN (Beijing)
}

1. Introduction. An arithmetic function $f(n)$ is said to be additive if it satisfies $f(a b)=f(a)+f(b)$, whenever $a$ and $b$ are coprime integers. For such a function we define

$$
A(x)=\sum_{p^{k} \leq x} \frac{f\left(p^{k}\right)}{p^{k}} \quad \text { and } \quad B(x)=\sum_{p^{k} \leq x} \frac{\left|f\left(p^{k}\right)\right|^{2}}{p^{k}} .
$$

The Turán-Kubilius inequality states that

$$
\sum_{n \leq x}|f(n)-A(x)|^{2} \leq c_{1} x B(x)
$$

for some absolute constant $c_{1}$, uniformly for all complex-valued additive functions $f(n)$ and real $x \geq 2$. (For the relevant literature on the TuránKubilius inequality, see Elliott [4]; in particular Chapter 4.)

Let $P(n)$ denote the largest prime factor of $n$ if $n>1$, and $P(1)=1$. Let $S(x, y)=\{n \mid 1 \leq n \leq x, P(n) \leq y\}$. In 1982, Alladi [1] obtained a Turán-Kubilius inequality for integers $n$ which have no large prime factors. More specifically, he showed that the inequality is valid uniformly for all strongly additive functions $f(n)$ and $n \in S(x, y)$, where $x, y$ satisfies $x \geq 20$, and $\exp \left\{(\log x)^{2 / 3}\right\} \leq y \leq x$. In [8], we further extended this range to $y \geq(\log x)^{1+\varepsilon}$.

In the present paper we derive a Turán-Kubilius inequality for (general) additive functions $f(n)$, where $n \in S(x, y)$.

We require uniform asymptotic estimates for $\Psi(x, y)$, where

$$
\Psi(x, y)=\sum_{n \leq x, P(n) \leq y} 1=|S(x, y)|
$$

1991 Mathematics Subject Classification: 11N30, 11K65.

Key words and phrases: additive function, Turán-Kubilius inequality, integers free of large prime factors. 
Recently Hildebrand [5] showed that the asymptotic formula

$$
\Psi(x, y)=x \varrho(u)\left\{1+O\left(\frac{\log (u+1)}{\log y}\right)\right\}, \quad u=\frac{\log x}{\log y},
$$

holds uniformly in the range

$$
x \geq 3, \quad \exp \left\{\left(\log _{2} x\right)^{5 / 3+\varepsilon}\right\} \leq y \leq x,
$$

where $\varepsilon>0$ is fixed, $\log _{2} x=\log \log x$ and the function $\varrho(u)$ is defined as the continuous solution of the system

$$
\begin{gathered}
\varrho(u)=1 \quad(0 \leq u \leq 1), \\
u \varrho^{\prime}(u)=-\varrho(u-1) \quad(u>1) .
\end{gathered}
$$

For a smaller range, (1.1) has been established by de Bruijn [3].

More recently, Hildebrand and Tenenbaum [6] showed that the asymptotic formula

$$
\Psi(x, y)=\frac{x^{\alpha} \zeta(\alpha, y)}{\alpha \sqrt{2 \pi \phi_{2}(\alpha, y)}}\left\{1+O\left(\frac{1}{u}\right)+O\left(\frac{\log y}{y}\right)\right\}
$$

holds uniformly in the range $x \geq y \geq 2$, where

$$
\begin{aligned}
\zeta(s, y) & =\prod_{p \leq y}\left(1-p^{-s}\right)^{-1}, \quad \operatorname{Re} s>0, \\
\phi(s, y) & =\log \zeta(s, y), \\
\phi_{k}(s, y) & =\frac{\partial^{k}}{\partial s^{k}} \phi(s, y), \quad k>0,
\end{aligned}
$$

and $\alpha=\alpha(x, y)$ is defined as the (unique) solution of the equation

$$
\phi_{1}(\alpha, y)+\log x=0 .
$$

Let $\xi=\xi(u)$ denote the unique positive solution of

$$
e^{\xi}=u \xi+1 \quad(u>1)
$$

and $\xi(u)=0(0 \leq u \leq 1)$. By the definition of $\xi(u)$ we readily deduce that

$$
\xi(u)=\log u+\log _{2} u+O(1) \text {. }
$$

Finally, we let

$$
\beta=\beta(x, y)=1-\frac{\xi(u)}{\log y}, \quad \beta_{k}=\beta\left(\frac{x}{p^{k}}, y\right), \quad u=\frac{\log x}{\log y} .
$$

We now state our main result.

THEOREM 1. For a complex-valued additive function $f$ we define

$$
H_{f}(x, y)=\sum_{p^{k} \leq x, p \leq y} \frac{f\left(p^{k}\right)}{p^{k} \varrho(u)} \varrho\left(u-\frac{\log p^{k}}{\log y}\right)\left(1-p^{-\beta_{k}}\right),
$$


and

$$
\Theta_{f}(x, y)=\sum_{p^{k} \leq x, p \leq y} \frac{\left|f\left(p^{k}\right)\right|^{2}}{p^{k} \varrho(u)} \varrho\left(u-\frac{\log p^{k}}{\log y}\right) .
$$

Then for all such $f$ and $x, y$ satisfying

$$
x \geq 3, \quad \exp \left\{(\log x)^{1 / 2+\varepsilon}\right\} \leq y \leq x,
$$

we have uniformly

$$
\sum_{n \in S(x, y)}\left|f(n)-H_{f}(x, y)\right|^{2} \ll \Psi(x, y) \Theta_{f}(x, y) .
$$

In addition, there is $u_{0} \geq 1$ such that for all $u \geq u_{0}$, the $\ll$ in (1.7) can be replaced by

$$
\leq \begin{cases}(1+o(1)), \text { as } x \rightarrow \infty, & \text { if } f>0, \\ (2+o(1)), \text { as } x \rightarrow \infty, & \text { for all other } f .\end{cases}
$$

In Theorem 1, we generalized the result of Alladi [1] to general additive functions. From the examples in Section 6 we see that the generalization is in a sense non-trivial.

From the proof of Theorem 1 in Section 3 we know that the exponent $1 / 2$ in the lower bound of (1.6) is best possible.

The function $H_{f}(x, y)$ in Theorem 1 can be replaced by

$$
\bar{H}_{f}(x, y)=\sum_{p^{k} \leq x, p \leq y} \frac{f\left(p^{k}\right)}{p^{k} \varrho(u)} \varrho\left(u-\frac{\log p^{k}}{\log y}\right) .
$$

In other words, we have the following

Corollary 1. For all complex-valued additive functions $f(n)$ and $x, y$ satisfying (1.6) we have uniformly

$$
\sum_{n \in S(x, y)}\left|f(n)-\bar{H}_{f}(x, y)\right|^{2} \ll \Psi(x, y) \Theta_{f}(x, y) .
$$

The dual of the last inequality is

TheOREM 2. Let $\left\{a_{n}\right\}$ be any sequence of complex numbers. Then for $x, y$ satisfying (1.6) we have uniformly

$$
\begin{array}{r}
\sum_{\substack{p^{k} \leq x \\
p \leq y}} \frac{p^{k} \varrho(u)}{\varrho\left(u-\frac{\log p^{k}}{\log y}\right)}\left|\sum_{\substack{n \in S(x, y) \\
p^{k} \| n}} a_{n}-\frac{\varrho\left(u-\frac{\log p^{k}}{\log y}\right)}{p^{k} \varrho(u)} \sum_{n \in S(x, y)} a_{n}\right|^{2} \\
\ll \Psi(x, y) \sum_{n \in S(x, y)}\left|a_{n}\right|^{2},
\end{array}
$$

where $p^{k} \| n$ means that $p^{k}$ divides $n$ ( $p$ prime), but $p^{k+1}$ does not. 
We omit the proof of this result, which is almost the same as that of Theorem 2 of Alladi [1].

As in [8] we shall further extend the range in Theorem 1. We now introduce some notations. Let $y$ be fixed and put $\alpha_{u}=\alpha\left(y^{u}, y\right)$ and

$$
\varrho^{*}(u)=\varrho^{*}\left(y^{u}, y\right):=\frac{y^{u\left(\alpha_{u}-1\right)} \zeta\left(\alpha_{u}, y\right)}{\alpha_{u} \sqrt{2 \pi \phi_{2}\left(\alpha_{u}, y\right)}}
$$

for $u \geq 1$ and $\varrho^{*}(u)=1$ for $0 \leq u \leq 1$. Then formula (1.3) becomes

$$
\Psi(x, y)=x \varrho^{*}(u)\left(1+O\left(\frac{1}{u}\right)+O\left(\frac{\log y}{y}\right)\right) .
$$

By part (ii) of Theorem 2 in [6], we see that

$$
\varrho^{*}(u)=\varrho(u) \exp \left\{O\left(\frac{1}{u}+\frac{\log (u+1)}{\log y}+u R_{\varepsilon}\right)\right\},
$$

where $R_{\varepsilon}=\exp \left\{-(\log y)^{3 / 5-\varepsilon}\right\}$ for $0<\varepsilon<1 / 2$.

TheOREM 3. For a complex-valued additive function, we define

$$
\begin{gathered}
H_{f}^{*}(x, y)=\sum_{p^{k} \leq x, p \leq y} \frac{f\left(p^{k}\right)}{p^{k} \varrho^{*}(u)} \varrho^{*}\left(u-\frac{\log p^{k}}{\log y}\right)\left(1-p^{-\alpha\left(x / p^{k}, y\right)}\right), \\
\Theta_{f}^{*}(x, y)=\sum_{p^{k} \leq x, p \leq y} \frac{\left|f\left(p^{k}\right)\right|^{2}}{p^{k} \varrho^{*}(u)} \varrho^{*}\left(u-\frac{\log p^{k}}{\log y}\right) .
\end{gathered}
$$

Then for all such $f$ and $x, y$ satisfying

$$
x \geq x_{0}, \quad(\log x)^{2+\varepsilon} \leq y \leq \exp \left\{\frac{\log x}{\log _{2} x}\right\},
$$

where $\varepsilon>0$ is fixed, we have uniformly

$$
\sum_{n \in S(x, y)}\left|f(n)-H_{f}^{*}(x, y)\right|^{2} \ll \Psi(x, y) \Theta_{f}^{*}(x, y) .
$$

Let

$$
\bar{H}_{f}^{*}(x, y)=\sum_{p^{k} \leq x, p \leq y} \frac{f\left(p^{k}\right)}{p^{k} \varrho^{*}(u)} \varrho^{*}\left(u-\frac{\log p^{k}}{\log y}\right) .
$$

COROLlary 2. For all complex-valued additive functions $f(n)$ and $x, y$ satisfying (1.13), we have uniformly

$$
\sum_{n \in S(x, y)}\left|f(n)-\bar{H}_{f}^{*}(x, y)\right|^{2} \ll \Psi(x, y) \Theta_{f}^{*}(x, y) .
$$


2. Some lemmas. To prove Theorem 1 we need the following lemmas.

Lemma 1. For $u \geq 1$ and $0 \leq t \ll 1$, we have uniformly

$$
\varrho(u-t)=\varrho(u) e^{t \xi(u)}\left(1+O\left(\frac{t}{u}\right)\right) .
$$

Proof. This is a slightly stronger form of [1, Lemma 3]; for the proof see [7].

Lemma 2. For $u \geq 1$ and $0 \leq t \leq u$, we have uniformly

$$
\varrho(u-t) \leq \varrho(u) e^{t \xi(u)}\left(1+O\left(u^{-1 / 3}\right)\right) .
$$

Proof. By Lemmas 2 and 3 of [7], we have uniformly for $u \geq 1$ and $0 \leq t \leq u^{2 / 3}$,

$$
\varrho(u-t)=\varrho(u) \exp \left\{t \xi(u)-\frac{t^{2}}{2} \xi^{\prime}(u)+\frac{t^{3}}{6} \xi^{\prime \prime}(u)\right\}\left(1+O\left(\frac{t}{u}\right)\right) .
$$

By the definition of $\xi(u)$ we have

$$
\xi^{\prime}(u)=\frac{\xi}{u \xi-u+1}=\frac{1}{u}\left(1+O\left(\frac{1}{\xi(u)}\right)\right) \quad(u>1)
$$

and

$$
\begin{aligned}
\xi^{\prime \prime}(u) & =-\frac{\xi^{3}\left(e^{\xi}\left(\xi^{2}-2 \xi+2\right)-2\right)}{\left(\xi e^{\xi}-e^{\xi}+1\right)^{3}} \\
& =-\frac{1}{u^{2}}\left(1+O\left(\frac{1}{\xi(u)}\right)\right) \quad(u>1),
\end{aligned}
$$

which implies that $\xi^{\prime}(u)>0$ and $\xi^{\prime \prime}(u)<0$ for $u>1$. From this and (2.2), (2.1) follows for $0 \leq t \leq u^{2 / 3}$.

We now turn our attention to the case $u^{2 / 3} \leq t \leq u-1$. By (3.9) of Alladi [1] we have

$$
\varrho(u)=\sqrt{\frac{\xi^{\prime}(u)}{2 \pi}} e^{\gamma-u \xi(u)+I(\xi(u))}\left(1+O\left(\frac{1}{u}\right)\right),
$$

where

$$
I(z)=\int_{0}^{z} \frac{e^{v}-1}{v} d v
$$

This implies

$$
\frac{\varrho(u-t)}{\varrho(u)}=\left(1+O\left(\frac{1}{u-t}\right)\right) \sqrt{\frac{\xi^{\prime}(u-t)}{\xi^{\prime}(u)}} \exp \{F(u, t)\},
$$


where

$$
F(u, t)=u \xi(u)-(u-t) \xi(u-t)+\int_{\xi(u)}^{\xi(u-t)} \frac{e^{v}-1}{v} d v
$$

From (2.3) and (2.6) we have in the range considered

$$
\frac{\varrho(u-t)}{\varrho(u)} \ll \sqrt{u} \exp \{F(u, t)\} .
$$

Thus to obtain (2.1) in the case $u^{2 / 3} \leq t \leq u-1$, it suffices to show

$$
F(u, t) \leq t \xi(u)-\frac{1}{2} t^{2} \xi^{\prime}(u) .
$$

Let

$$
G(u, t)=F(u, t)-t \xi(u)+\frac{1}{2} t^{2} \xi^{\prime}(u) .
$$

By (2.7), (2.3), (2.4) and the definition of $\xi(u)$ we find that $\left(\partial^{2} / \partial t^{2}\right) G(u, t)$ $<0,(\partial / \partial t) G(u, t)<0$ and hence $G(u, t)<0$ for $t>0$. Thus (2.9) follows.

Finally, we consider the case $u-1 \leq t \leq u$. It is well known that

$$
\varrho(u)=e^{-u \xi(u)+u+O(u / \log u)} .
$$

This implies (2.1).

The proof of Lemma 2 is complete.

Lemma 3. For $u \geq u_{0}$ ( $u_{0}$ is a sufficiently large absolute constant), $0 \leq t \leq s, s \geq \frac{1}{3} u, t+s \leq u$, we have uniformly

$$
\varrho(u) \varrho(u-t-s) \ll \varrho(u-t) \varrho(u-s) .
$$

Proof. If $t \leq \sqrt{u}$ and $t+s \leq u-1$, from (2.2)-(2.4) we have

$$
\varrho(u-t)=\varrho(u) e^{t \xi(u)}\left(1+O\left(\frac{t+t^{2}}{u}\right)\right) .
$$

Lemma 2 implies

$$
\varrho(u-t-s) \ll \varrho(u-s) e^{t \xi(u-s)} \ll \varrho(u-s) e^{t \xi(u)} .
$$

From these two estimates, (2.10) follows for $0 \leq t \leq \sqrt{u}$.

If $t>\sqrt{u}$ and $t+s \leq u-1$, we let

$$
P(u, t, s)=\varrho(u) \varrho(u-t-s) /(\varrho(u-t) \varrho(u-s)) .
$$

By (2.5) and (2.3) we have

$$
\begin{aligned}
& P(u, t, s) \\
& \quad=\left(1+O\left(\frac{1}{u-t-s}\right)\right) \sqrt{\frac{\xi^{\prime}(u) \xi^{\prime}(u-t-s)}{\xi^{\prime}(u-t) \xi^{\prime}(u-s)}} \exp \{F(u, t, s)\} \\
& \quad \leq u \exp \{F(u, t, s)\}
\end{aligned}
$$


where

$$
\begin{aligned}
& F(u, t, s) \\
& =(u-t) \xi(u-t)+(u-s) \xi(u-s)-u \xi(u)-(u-t-s) \xi(u-t-s) \\
& \quad+\int_{\xi(u-t)}^{\xi(u-t-s)} \frac{e^{v}-1}{v} d v-\int_{\xi(u)}^{\xi(u-s)} \frac{e^{v}-1}{v} d v .
\end{aligned}
$$

Thus to obtain (2.10) in the case considered it suffices to show

$$
F(u, t, s) \leq e^{-t s \xi^{\prime}(u)} .
$$

Let $G(\lambda)=F(u, \lambda t, \lambda s)+\lambda^{2} t s \xi^{\prime}(u), 0 \leq \lambda \leq 1$. We proceed as in the proof of (2.9) to get $G(\lambda)<0$ for $0<\lambda \leq 1$. From this, (2.12) follows.

Finally, we consider the case $u-1 \leq t+s \leq u$. We put $t_{1}=t-a$, $s_{1}=s-b$, with $a \geq 0, b \geq 0$, and $a+b=1$. Then $t_{1}+s_{1} \leq u-1$, and

$$
\varrho(u-t) \geq \varrho\left(u-t_{1}\right), \quad \varrho(u-s) \geq \varrho\left(u-s_{1}\right),
$$

since $\varrho(u)$ is decreasing. By the above result in the case $t+s \leq u-1$, we obtain

$$
P(u, t, s) \ll \frac{\varrho(u) \varrho\left(u-t_{1}-s_{1}\right)}{\varrho\left(u-t_{1}\right) \varrho\left(u-s_{1}\right)} \ll 1 .
$$

The proof of Lemma 3 is now complete.

Remark. For $u \geq u_{0}, 0 \leq t \leq s \leq \frac{1}{3} u$, by Lemma 4 of [1], we have

$$
\varrho(u) \varrho(u-t-s) \leq \varrho(u-t) \varrho(u-s) .
$$

If $1 \leq u \leq u_{0}$, we have $\varrho(u-t) / \varrho(u) \geq 1$, since $\varrho(u)$ is decreasing, and $\varrho(u-t-s) / \varrho(u-s) \ll 1$, by Lemma 2 . So we have $\varrho(u) \varrho(u-t-s) \ll$ $\varrho(u-t) \varrho(u-s)$ in the ranges considered.

Collecting the above estimates yields

$\varrho(u) \varrho(u-t-s) \ll \varrho(u-t) \varrho(u-s) \quad$ for $u \geq 1, t \geq 0, s \geq 0$ and $t+s \leq u$.

Let

$$
H(x, y)=\sum_{p^{k} \leq x, p \leq y} \frac{1}{p^{k} \varrho(u)} \varrho\left(u-\frac{\log p^{k}}{\log y}\right) .
$$

Lemma 4. For $x$, y satisfying (1.6) we have uniformly

$$
H(x, y)=\operatorname{li}(u \xi(u))-\log _{2} u+\log _{2} y+O(1) .
$$

Pr o of. By Theorem 3 of [1] we have

$$
\sum_{p \leq y} \frac{1}{p \varrho(u)} \varrho\left(u-\frac{\log p}{\log y}\right)=\operatorname{li}(u \xi(u))-\log _{2} u+\log _{2} y+O(1) .
$$


By Lemma 2 we get

$$
\sum_{p^{k} \leq x, p \leq y, k \geq 2} \frac{1}{p^{k} \varrho(u)} \varrho\left(u-\frac{\log p^{k}}{\log y}\right) \ll \sum_{p \leq y} \frac{1}{p^{2}} e^{2(\log p) \xi(u) / \log y} \ll 1 .
$$

Thus (2.13) follows.

Lemma 5. For any fixed positive integer $a$ and $x$, $y$ satisfying (1.6) we have uniformly

$$
\sum_{p^{k} \leq x, p \leq y} \frac{1}{p^{k} \varrho(u)} \varrho\left(u-\frac{\log p^{k}}{\log y}\right)\left(\frac{\log p^{k}}{\log y}\right)^{a} \ll u .
$$

Proof. By Lemma 2 and the prime number theorem, the left-hand side of $(2.14)$ is

$$
\begin{aligned}
& \ll \sum_{p \leq y} \frac{1}{p} e^{(\log p) \xi(u) / \log y}\left(\frac{\log p}{\log y}\right)^{a} \\
& \ll \int_{2}^{y} e^{(\log z) \xi(u) / \log y}\left(\frac{\log z}{\log y}\right)^{a} \frac{d z}{z \log z} \\
& \ll \frac{1}{\xi(u)^{a}} \int_{\eta \log 2}^{\xi(u)} e^{w} w^{a-1} d w \ll u,
\end{aligned}
$$

where $\eta=\xi(u) / \log y$.

Lemma 6. For $x$, y satisfying (1.6) we have uniformly

$$
\sum^{\prime} \frac{f\left(p^{k}\right)}{p^{k} \varrho(u)} \varrho\left(u-\frac{\log p^{k}}{\log y}\right) \ll \sqrt{\Theta_{f}(x, y)}(\log x)^{-3},
$$

where $\sum^{\prime}$ denotes a sum over integers $p, k$ with the restrictions $p^{k} \leq x$, $p \leq y$ and $k>(\log x) /(3 \log p)$.

Proof. Applying the Cauchy-Schwarz inequality, the left-hand side of $(2.15)$ is

$$
\ll \sqrt{\Theta_{f}(x, y)}\left\{\sum^{\prime} \frac{1}{p^{k} \varrho(u)} \varrho\left(u-\frac{\log p^{k}}{\log y}\right)\right\}^{1 / 2} .
$$

By Lemma 2, the last sum is

$$
\ll \sum_{p \leq y} p^{-k_{0}} e^{k_{0}(\log p) \xi(u) / \log y} \ll(\log x)^{-3},
$$

where $k_{0}=[\log x /(3 \log p)]$. This provides the desired estimate.

Lemma 7. For $x$, y satisfying (1.6) we have uniformly

$$
\sum^{\prime \prime} \frac{f\left(p^{k}\right) f\left(q^{l}\right)}{p^{k} q^{l} \varrho(u)} \varrho\left(u-\frac{\log p^{k}}{\log y}-\frac{\log q^{l}}{\log y}\right) \ll \Theta_{f}(x, y)(\log x)^{-3},
$$


where $\sum^{\prime \prime}$ denotes a sum over integers $p, q, k, l$ with the restrictions $p^{k} q^{l} \leq$ $x, p \leq y, q \leq y$, and $k \geq(\log x) /(3 \log p)($ or $l \geq(\log x) /(3 \log q))$.

Proof. By Lemmas 3 and 6, the left-hand side of (2.16) is

$$
\ll H_{f}(x, y) \sqrt{\Theta_{f}(x, y)}(\log x)^{-4} .
$$

The Cauchy-Schwarz inequality and Lemma 4 give

$$
H_{f}(x, y) \ll \sqrt{\Theta_{f}(x, y)\left(u+\log _{2} y\right)},
$$

which completes the proof of Lemma 7 .

3. Proofs of Theorem 1 and Corollary 1. We may suppose without loss of generality that $f \geq 0$. If $f$ is any real-valued additive function, we define an additive function $f_{j}(n), j=1,2$ by

$$
\begin{aligned}
& f_{1}\left(p^{k}\right)= \begin{cases}f\left(p^{k}\right) & \text { if } f\left(p^{k}\right)>0, \\
0 & \text { otherwise }\end{cases} \\
& f_{2}\left(p^{k}\right)= \begin{cases}-f\left(p^{k}\right) & \text { if } f\left(p^{k}\right)<0, \\
0 & \text { otherwise }\end{cases}
\end{aligned}
$$

Then we have

$$
\left(f(n)-H_{f}(x, y)\right)^{2} \leq 2\left(f_{1}(n)-H_{f_{1}}(x, y)\right)^{2}+2\left(f_{2}(n)-H_{f_{2}}(x, y)\right)^{2}
$$

and so the desired estimate for $f$ follows from that for $f_{1}$ and $f_{2}$. Similarly if $f$ is complex-valued, we obtain the result by breaking it into its real and imaginary parts.

By the additivity of $f(n),(1.1)$ and Lemma 1 we have

$$
\begin{aligned}
\sum_{n \in S(x, y)} f(n)= & \sum_{p^{k} \leq x, p \leq y} f\left(p^{k}\right)\left\{\Psi\left(\frac{x}{p^{k}}, y\right)-\Psi\left(\frac{x}{p^{k+1}}, y\right)\right\} \\
= & \Psi(x, y) H_{f}(x, y)\left(1+O\left(\frac{\log (u+1)}{\log y}\right)\right) \\
& +O\left(R_{1}\right)+O\left(R_{2}\right)
\end{aligned}
$$

where

$$
\begin{aligned}
& R_{1}=\Psi(x, y) \sum_{p \leq y, k \leq k_{0}} \frac{f\left(p^{k}\right)}{p^{k} \varrho(u)} \varrho\left(u-\frac{\log p^{k}}{\log y}\right) p^{-\beta_{k}}\left(\frac{\log p}{\log x}\right), \\
& R_{2}=\Psi(x, y) \sum_{p^{k} \leq x, p \leq y, k>k_{0}} \frac{f\left(p^{k}\right)}{p^{k} \varrho(u)} \varrho\left(u-\frac{\log p^{k}}{\log y}\right) p^{-\beta_{k}}
\end{aligned}
$$

$\left(k_{0}=[\log x /(3 \log p)]\right)$. Applying the Cauchy-Schwarz inequality we get

$$
R_{1} \ll \Psi(x, y) \sqrt{\Theta_{f}(x, y)}(\log x)^{-1} .
$$


By Lemma 6 we have

$$
R_{2} \ll \Psi(x, y) \sqrt{\Theta_{f}(x, y)}(\log x)^{-1} .
$$

From (3.1)-(3.3), (2.17) and (1.6) we further have

$$
\begin{aligned}
W:= & \sum_{n \in S(x, y)}\left\{f(n)-H_{f}(x, y)\right\}^{2} \\
= & \sum_{n \in S(x, y)} f^{2}(n)-2 H_{f}(x, y) \sum_{n \in S(x, y)} f(n) \\
& +\Psi(x, y) H_{f}(x, y)^{2} \\
= & \sum_{n \in S(x, y)} f^{2}(n)-\Psi(x, y) H_{f}(x, y)^{2} \\
& +O\left(\Psi(x, y) \Theta_{f}(x, y)(\log x)^{-\varepsilon}\right) .
\end{aligned}
$$

We also have

$$
\begin{aligned}
\sum_{n \in S(x, y)} f^{2}(n) \leq & \sum_{\substack{p^{k} q^{l} \leq x \\
p \leq y, q \leq y}} f\left(p^{k}\right) f\left(q^{l}\right)\left\{\Psi\left(\frac{x}{p^{k} q^{l}}, y\right)-\Psi\left(\frac{x}{p^{k+1} q^{l}}, y\right)\right. \\
& \left.-\Psi\left(\frac{x}{p^{k} q^{l+1}}, y\right)+\Psi\left(\frac{x}{p^{k+1} q^{l+1}}, y\right)\right\} \\
& +\sum_{\substack{p^{k} \leq x, p \leq y \\
=}} f^{2}\left(p^{k}\right)\left\{\Psi\left(\frac{x}{p^{k}}, y\right)-\Psi\left(\frac{x}{p^{k+1}}, y\right)\right\} \\
= & W_{1}+W_{2}, \quad \text { say . }
\end{aligned}
$$

Put $\log p^{k} / \log y=t, \log q^{l} / \log y=s$,

$$
\beta\left(x / p^{k}, y\right)=\beta_{k}, \quad \beta\left(x / q^{l}, y\right)=\beta_{l}, \quad \beta\left(x /\left(p^{k} q^{l}\right), y\right)=\beta_{k l} .
$$

By (1.1), and Lemmas 1 and 7 we obtain

$$
\begin{aligned}
W_{1}= & \Psi(x, y) \sum^{*} \frac{f\left(p^{k}\right) f\left(q^{l}\right)}{p^{k} q^{l} \varrho(u)} \varrho(u-t-s)\left(1-p^{-\beta_{k l}}\right)\left(1-q^{-\beta_{k l}}\right) \\
& \times\left(1+O\left(\frac{\log (u+1)}{\log y}\right)\right) \\
+ & O\left(\Psi(x, y) \sum^{*} \frac{f\left(p^{k}\right) f\left(q^{l}\right)}{p^{k} q^{l} \varrho(u)} \varrho(u-t-s)\right. \\
& \left.\times\left(p^{-\beta_{k l}}\left(\frac{\log p}{\log x}\right)+q^{-\beta_{k l}}\left(\frac{\log q}{\log x}\right)\right)\right) \\
& +O\left(\Psi(x, y) \Theta_{f}(x, y)(\log x)^{-\varepsilon}\right) \\
= & W_{1}^{\prime}+O\left(R_{3}\right)+O(E), \text { say },
\end{aligned}
$$


where $\sum^{*}$ denotes a sum over integers $p, q, k, l$ with the restrictions $p \leq y$, $q \leq y, k \leq \log x /(3 \log p)$ and $l \leq \log x /(3 \log q)$.

We first consider the case $u \geq u_{0}$. By Lemma 4 of [1] we get

$$
\begin{aligned}
W_{1}^{\prime} \leq \Psi(x, y) \sum^{*} & \frac{f\left(p^{k}\right) f\left(q^{l}\right)}{p^{k} q^{l}} \frac{\varrho(u-t) \varrho(u-s)}{\varrho(u)^{2}} \\
& \times\left(1-p^{-\beta_{k}}\right)\left(1-q^{-\beta_{l}}\right)\left(1+O\left(\frac{\log (u+1)}{\log y}\right)\right) \\
+ & O\left(\Psi(x, y) \sum^{*} \frac{f\left(p^{k}\right) f\left(q^{l}\right)}{p^{k} q^{l}} \frac{\varrho(u-t) \varrho(u-s)}{\varrho(u)^{2}}\right. \\
& \left.\times\left(\left(p^{-\beta_{k}}-p^{-\beta_{k l}}\right)+\left(q^{-\beta_{l}}-q^{-\beta_{k l}}\right)\right)\right) \\
\leq \Psi(x, y) H_{f}(x, y)^{2}(1+ & O(\log (u+1) / \log y))+O\left(R_{4}\right), \quad \text { say . }
\end{aligned}
$$

It is easy to prove that

$$
p^{-\beta_{k}}-p^{-\beta_{k l}} \ll p^{-\beta_{k}}\left(\frac{s \log p}{u \log y}\right) .
$$

From this, applying the Cauchy-Schwarz inequality and Lemma 5 we have

$$
R_{4} \ll \Psi(x, y)(\log x)^{-1} \sqrt{\Theta_{f}(x, y)} \cdot \sqrt{u \Theta_{f}(x, y)} \ll E .
$$

Similarly, we have

$$
R_{3} \ll \Psi(x, y)(\log x)^{-1} H_{f}(x, y) \cdot \sqrt{\Theta_{f}(x, y)} \ll E .
$$

From (3.6)-(3.9) and (2.17) we obtain

$$
W_{1} \leq \Psi(x, y) H_{f}(x, y)^{2}+O(E),
$$

where $E$ is defined as in (3.6).

We next turn to $W_{2}$. In view of (1.1) and (1.6), we get

$$
\begin{aligned}
W_{2} & \leq \sum_{p^{k} \leq x, p \leq y} f^{2}\left(p^{k}\right) \Psi\left(\frac{x}{p^{k}}, y\right) \\
& =\Psi(x, y) \sum_{p^{k} \leq x, p \leq y} \frac{f^{2}\left(p^{k}\right)}{p^{k} \varrho(u)} \varrho\left(u-\frac{\log p^{k}}{\log y}\right)\left(1+O\left(\frac{\log (u+1)}{\log y}\right)\right) \\
& =\Psi(x, y) \Theta_{f}(x, y)+O(E) .
\end{aligned}
$$

Collecting (3.4), (3.5), (3.10) and (3.11) yields

$$
W \leq \Psi(x, y) \Theta_{f}(x, y)\left(1+O\left((\log x)^{-\varepsilon}\right)\right) .
$$

Now we turn our attention to the case $u \leq u_{0}$. From the remark of Lemma 3, we have

$$
\varrho(u) \varrho(u-t-s) \ll \varrho(u-t) \varrho(u-s) .
$$


By the same argument as before, we have

$$
W \ll \Psi(x, y) \Theta_{f}(x, y) .
$$

The proof of Theorem 1 is now complete.

Remark. In (3.4) there is an error term $O\left(\Psi(x, y) \Theta_{f}(x, y)\left(u+\log _{2} y\right)\right.$ $\times(\log (u+1) / \log y))$, where the factor $O(\log (u+1) / \log y)$ comes from the error term in (1.1), which is best possible. From this we see that the exponent $1 / 2$ in lower bound (1.6) is best possible.

Proof of Corollary 1 . We may suppose without loss of generality that $f \geq 0$. We have

$$
H_{f}(x, y)=\bar{H}_{f}(x, y)-\Delta(x, y),
$$

where

$$
\Delta(x, y)=\sum_{p^{k} \leq x, p \leq y} \frac{f\left(p^{k}\right)}{p^{k} \varrho(u)} \varrho\left(u-\frac{\log p^{k}}{\log y}\right) p^{-\beta_{k}} .
$$

By (3.1)-(3.3) and (3.14) we have

$$
\sum_{n \in S(x, y)} f(n)=\Psi(x, y) \bar{H}_{f}(x, y)\left(1+O\left(\frac{\log (u+1)}{\log y}\right)\right)-\Psi(x, y) \Delta(x, y) .
$$

As for $W$ of (3.4) we obtain similarly

$$
\begin{aligned}
\bar{W}:= & \sum_{n \in S(x, y)}\left\{f(n)-\bar{H}_{f}(x, y)\right\}^{2} \\
= & \sum_{n \in S(x, y)} f^{2}(n)-\Psi(x, y) \bar{H}_{f}(x, y)^{2} \\
& +2 \Psi(x, y) \bar{H}_{f}(x, y) \Delta(x, y)+O\left(\Psi(x, y) \Theta_{f}(x, y)(\log x)^{-\varepsilon}\right) .
\end{aligned}
$$

In view of (3.5), (3.10), (3.11) and (3.13) we have

$$
\begin{aligned}
& \sum_{n \in S(x, y)} f^{2}(n) \\
& \quad \leq \Psi(x, y) \bar{H}_{f}(x, y)^{2}-2 \Psi(x, y) \bar{H}_{f}(x, y) \Delta(x, y) \\
& \quad+\Psi(x, y) \Delta(x, y)^{2}+\Psi(x, y) \Theta_{f}(x, y)\left(1+O\left((\log x)^{-\varepsilon}\right)\right) .
\end{aligned}
$$

Applying the Cauchy-Schwarz inequality we have

$$
\Delta(x, y)^{2} \leq \Theta_{f}(x, y) \sum_{p^{k} \leq x, p \leq y} \frac{1}{p^{k} \varrho(u)} \varrho\left(u-\frac{\log p^{k}}{\log y}\right) p^{-2 \beta_{k}} .
$$

Denoting by $\Sigma$ the sum on the right-hand side of (3.17) we may write 


$$
\begin{aligned}
\Sigma & =\sum_{p \leq Y, k \leq \xi(u)}+\sum_{p^{k} \leq x, p \leq Y, k>\xi(u)}+\sum_{p^{k} \leq x, Y<p \leq y} \\
& =\Sigma_{1}+\Sigma_{2}+\Sigma_{3}, \quad \text { say },
\end{aligned}
$$

where $Y=\exp \sqrt{\log y}$. By Lemma 2 we have

$$
\begin{aligned}
\Sigma_{1} & \leq \sum_{p \leq Y, k \leq \xi(u)} \frac{1}{p^{k+2}} e^{(k+2)(\log p) \xi(u) / \log y}\left(1+O\left(\frac{1}{u^{1 / 3}}\right)\right) \\
& \leq \sum_{p} \sum_{k=1}^{\infty} \frac{1}{p^{k+2}}\left(1+O\left(\frac{1}{u^{1 / 3}}\right)\right) \\
& =C_{0}\left(1+O\left(u^{-1 / 3}\right)\right), \quad \text { say },
\end{aligned}
$$

where $\sum_{p}$ denotes a sum taken over all prime numbers.

It is easy to prove that

$$
\Sigma_{2}+\Sigma_{3} \ll u^{-1}
$$

From this and (3.17)-(3.19) we obtain

$$
\Delta(x, y)^{2} \leq C_{0}\left(1+O\left(u^{-1 / 3}\right)\right) \Theta_{f}(x, y) .
$$

Combining (3.15), (3.16) and (3.20) completes the proof of Corollary 1.

Rem ark. For strongly additive functions, Alladi [1] obtained a TuránKubilius inequality for $S(x, y)$. The result can be deduced from Theorem 1, the argument is almost the same as that of Corollary 1.

4. Preliminaries to the proof of Theorem 3. In order to prove Theorem 3, we need some lemmas.

Lemma 8. For $x \geq y \geq 2$ and $1 \leq d \leq y$ we have uniformly

$$
\Psi\left(\frac{x}{d}, y\right)=\Psi(x, y) d^{-\alpha(x, y)}\left(1+O\left(\frac{1}{u}+\frac{\log y}{y}\right)\right) .
$$

Proof. See [6, Theorem 3].

Lemma 9. For $u \geq 2,0 \leq t \leq u, y \geq y_{0}$ and $y \geq u^{1+\varepsilon}$ where $y_{0}$ is a sufficiently large absolute constant, and $\varepsilon>0$ is fixed, we have uniformly

$$
\varrho^{*}(u-t) \ll \varrho^{*}(u) \exp \left\{t\left(1-\alpha_{u}\right) \log y\right\} .
$$

Pr o of. We first consider the case $t \leq u-1$. By (1.8) we have

$$
\frac{\varrho^{*}(u-t)}{\varrho^{*}(u)}=\frac{\alpha_{u}}{\alpha_{u-t}} \sqrt{\frac{\phi_{2}\left(\alpha_{u}, y\right)}{\phi_{2}\left(\alpha_{u-t}, y\right)}} \exp \left\{F^{*}(u, t)\right\},
$$

where

(4.2) $F^{*}(u, t):=\left(u \alpha_{u-t}-u \alpha_{u}-t \alpha_{u-t}+t\right) \log y+\phi\left(\alpha_{u-t}, y\right)-\phi\left(\alpha_{u}, y\right)$. 
From (1.4) we have

$$
\phi_{1}\left(\alpha_{u}, y\right)+u \log y=0 .
$$

Differentiating (4.3) with respect to $u$ and using Lemma 4 of [6] then yields

$$
\alpha_{u}^{\prime}=-\log y / \phi_{2}\left(\alpha_{u}, y\right)<0,
$$

hence $\alpha_{u}$ in decreasing, so that $\alpha_{u} / \alpha_{u-t} \leq 1$. By Lemma 4 of [6] we have

$$
\phi_{2}\left(\alpha_{u}, y\right) / \phi_{2}\left(\alpha_{u-t}, y\right) \ll U^{2}(u, t),
$$

where $U(u, t)=1$ if $|t| \leq u / 2$, and $=\sqrt{u}$, otherwise. From this and (4.1) we get

$$
\varrho^{*}(u-t) / \varrho^{*}(u) \ll U(u, t) \exp \left\{F^{*}(u, t)\right\} .
$$

By (7.19) of $[6]$ and (4.4) we have

$$
\alpha_{u}^{\prime}=-\frac{\xi^{\prime}(u)}{\log y}\left(1+O\left(R_{\varepsilon}+(u \log y)^{-1}\right)\right),
$$

where $R_{\varepsilon}=\exp \left\{(-\log y)^{3 / 5-\varepsilon}\right\}$.

Therefore to prove the lemma for $t \leq u-1$, it suffices to show

$$
F^{*}(u, t)<t\left(1-\alpha_{u}\right) \log y+\frac{1}{2} t^{2} \alpha_{u}^{\prime} \log y \quad(0<t \leq u-1) .
$$

By the same argument as in the proof of (2.9), and using Lemma 4 of [6], (4.6) is derived.

If $u-1 \leq t \leq u$, by (7.8) of [6] and (1.10) we have

$$
\begin{aligned}
& \varrho^{*}(u) \exp \left\{t\left(1-\alpha_{u}\right) \log y\right\} \geq \varrho^{*}(u) \exp \left\{(u-1) \xi(u)+O\left(u R_{\varepsilon}\right)\right\} \\
& \quad \geq \exp \left\{u+O\left(\frac{u}{\log u}\right)+O\left(\frac{\log (u+1)}{\log y}+u R_{\varepsilon}\right)\right\} \gg 1 .
\end{aligned}
$$

This completes the proof of Lemma 9 .

Lemma 10. For $u \geq u_{0}, t \geq 0, s \geq 0, t+s \leq u-1$, and $y \geq u^{1+\varepsilon}(\varepsilon>0$ is fixed), we have uniformly

$$
\frac{\varrho^{*}(u) \varrho^{*}(u-t-s)}{\varrho^{*}(u-t) \varrho^{*}(u-s)} \leq 1+O\left(\frac{\min (t, s)}{u}\right) .
$$

Pro of. Denoting the left-hand side of the above formula by $Q(u, t, s)$, we have by (1.8),

$$
Q(u, t, s)=\frac{\alpha_{u-t} \alpha_{u-s}}{\alpha_{u} \alpha_{u-t-s}} \sqrt{\frac{\alpha_{u}^{\prime} \alpha_{u-t-s}^{\prime}}{\alpha_{u-t}^{\prime} \alpha_{u-s}^{\prime}}} \exp \{R(u, t, s)\},
$$

where

$$
\begin{aligned}
R(u, t, s)= & u \alpha_{u} \log y+(u-t-s) \alpha_{u-t-s} \log y \\
& -(u-t) \alpha_{u-t} \log y-(u-s) \alpha_{u-s} \log y \\
& +\phi\left(\alpha_{u}, y\right)+\phi\left(\alpha_{u-t-s}, y\right)-\phi\left(\alpha_{u-t}, y\right)-\phi\left(\alpha_{u-s}, y\right) .
\end{aligned}
$$


It is easy to prove that

$$
R(u, t, s) \leq t s \alpha_{u}^{\prime} \log y
$$

and

$$
\alpha_{u-t} \alpha_{u-s}-\alpha_{u} \alpha_{u-t-s} \leq 0 .
$$

We also have $\alpha_{u-t}^{\prime}<\alpha_{u}^{\prime}<0$, and hence $\alpha_{u}^{\prime} / \alpha_{u-s}^{\prime} \leq 1$. From this, (4.7), (4.9) and (4.10), we obtain

$$
Q(u, t, s) \leq \sqrt{\alpha_{u-t-s}^{\prime} / \alpha_{u-t}^{\prime}} \exp \left\{t s \alpha_{u}^{\prime} \log y\right\} .
$$

Next we will give a relatively sharp estimate for $Q(u, t, s)$. We may suppose without loss of generality that $t \geq s$.

Let

$$
\begin{aligned}
D & :=\{(t, s) \mid t \geq s>0, t+s \leq u-1\}, \\
D_{1} & :=\left\{(t, s) \mid t \geq s, t s>u^{4 / 3}, t+s \leq u-1\right\}, \\
D_{2} & :=\left\{(t, s) \mid t \geq s>0, t s \leq u^{4 / 3}, t \leq 6 u / 7\right\}, \\
D_{3} & :=\left\{(t, s) \mid s>0, t s \leq u^{4 / 3}, 6 u / 7<t \leq u-1-s\right\},
\end{aligned}
$$

where $u \geq u_{0}$. It is obvious that $D=D_{1} \cup D_{2} \cup D_{3}$.

In the case $(t, s) \in D_{1}$, we have $\sqrt{\alpha_{u-t-s}^{\prime} / \alpha_{u-t}^{\prime}} \ll \sqrt{u}$ by (6.6) of [6]. From this, (4.11), (4.5) and (2.3) we get

$$
Q(u, t, s) \ll \sqrt{u} \exp \left\{-\frac{1}{2} u^{1 / 3}\right\} \ll u^{-4} \quad \text { for }(t, s) \in D_{1} .
$$

In the case $(t, s) \in D_{2}$, by Taylor's formula, we have $\sqrt{\alpha_{u-t-s}^{\prime} / \alpha_{u-t}^{\prime}}=$ $1+O(s / u)$. From this and (4.11) we have

$$
Q(u, t, s) \leq 1+O(s / u) \quad \text { for }(t, s) \in D_{2} .
$$

Finally, in the case $(t, s) \in D_{3}$, we have $\alpha_{u}^{\prime} \log y \leq-7 u / 8$ by (4.5). Noting that $t \geq 6 u / 7$, it then follows that

$$
Q(u, t, s) \leq \sqrt{\xi^{\prime}(u-t-s) / \xi^{\prime}(u-t)} e^{-(3 / 4) s}=: N(s), \quad \text { say } .
$$

Differentiating the above equation, we find

$$
N^{\prime}(s)=\frac{-e^{-(3 / 4) s}}{2 \sqrt{\xi^{\prime}(u-t) \xi^{\prime}(u-t-s)}}\left(\xi^{\prime \prime}(u-t-s)+\frac{3}{2} \xi^{\prime}(u-t-s)\right) .
$$

If we assume for the moment that

$$
\xi^{\prime \prime}(u)+a \xi^{\prime}(u)>0 \quad \text { for } u>1, a \geq 4 / 3,
$$

then it follows that $N^{\prime}(s)<0$ for $s>0$, hence $N(s)<N(0)=1$. From this and (4.14) we obtain

$$
Q(u, t, s) \leq 1 \quad \text { for }(t, s) \in D_{3},
$$

and hence the assertion of Lemma 10. 
It remains to prove the inequality (4.15). By (2.3), (2.4) and definition of $\xi(u)$ we find

$$
\xi^{\prime \prime}(u)+a \xi^{\prime}(u)=\frac{L(\xi)}{\xi\left(e^{\xi}-u\right)^{3}}, \quad \text { say },
$$

where

$$
L(\xi):=e^{2 \xi}\left(a \xi^{2}-2 a \xi+a\right)+e^{\xi}\left(-\xi^{3}+2 \xi^{2}+2 a \xi-2 \xi-2 a\right)+2 \xi+a .
$$

We also have $\xi\left(e^{\xi}-u\right)=\left(\xi e^{\xi}-e^{\xi}+1\right)>0$ for $\xi>0$. To obtain (4.15) it therefore suffices to show

$$
L(\xi)>0 \quad \text { for } \xi>0 .
$$

A simple calculation gives

$$
\begin{gathered}
L^{(n)}(\xi) \geq 0 \quad \text { for } \xi=0, n=0,1,2, \\
\frac{d^{n}}{d \xi^{n}}\left(L^{\prime \prime}(\xi) e^{-\xi}\right) \geq 0 \quad \text { for } \xi=0, n=1,2,3, \\
\frac{d^{4}}{d \xi^{4}}\left(L^{\prime \prime}(\xi) e^{-\xi}\right)=e^{\xi}\left(4 a \xi^{2}+32 a \xi+46 a\right)>0 \quad \text { for } \xi>0 .
\end{gathered}
$$

These inequalities imply (4.17), and hence (4.15). Combining (4.12), (4.13) and (4.16) completes the proof of Lemma 10.

Let

$$
H^{*}(x, y)=\sum_{p^{k} \leq x, p \leq y} \frac{1}{p^{k} \varrho^{*}(u)} \varrho^{*}\left(u-\frac{\log p^{k}}{\log y}\right) .
$$

LeMma 11. For $x$, $y$ satisfying

$$
x \geq x_{0}, \quad(\log x)^{2+\varepsilon} \leq y \leq x,
$$

we have uniformly

$$
H^{*}(x, y)=u+\log _{2} y+O\left(\frac{u}{\log u}\right)+O\left(u \exp \left\{-(\log y)^{3 / 5-\varepsilon}\right\}\right) .
$$

Proof. We may write

$$
H^{*}(x, y)=\sum_{p \leq y, k=1}+\sum_{p^{k} \leq x, p \leq y, k \geq 2}=\Sigma_{1}+\Sigma_{2} .
$$

By the same argument as in [1], we have

$$
\Sigma_{1}=u+\log _{2} y+O\left(\frac{u}{\log u}\right)+O\left(u \exp \left\{-(\log y)^{3 / 5-\varepsilon}\right\}\right) .
$$

By Lemma 9 we obtain

$$
\Sigma_{2} \ll \sum_{p^{k} \leq x, p \leq y, k \geq 2} p^{-k \alpha_{u}} \ll \sum_{p \leq y} p^{-2 \alpha_{u}} .
$$


By (7.8) of [6] we have $1-\alpha_{n} \leq 1 /(2+\varepsilon / 2)$, for $x, y$ satisfying (4.18) and $x_{0}$ sufficiently large. This implies $\Sigma_{2} \ll 1$.

This completes the proof of Lemma 11.

Moreover, we also need the following results, which can be established in the same way as Lemma 11.

LEMma 12. For $x$, y satisfying (4.18) we have uniformly

$$
\Sigma_{A}:=\sum_{p^{k} \leq x, p \leq y} \frac{1}{p^{k} \varrho^{*}(u)} \varrho^{*}\left(u-\frac{\log p^{k}}{\log y}\right)\left(\frac{\log p^{k}}{\log y}\right)^{a} \ll u,
$$

where $a$ is any fixed positive integer.

Lemma 13. For $x, y$ satisfying (4.18) we have uniformly

$$
\Sigma_{B}:=\sum_{p^{k} \leq x, p \leq y, k>k_{0}} \frac{1}{p^{k} \varrho^{*}(u)} \varrho^{*}\left(u-\frac{\log p^{k}}{\log y}\right) \ll(\log x)^{-3},
$$

where $k_{0}$ is defined as in Lemma 6.

5. Proofs of Theorem 3 and Corollary 2. The proof of Theorem 3 is somewhat similar to the proof of Theorem 1. We may suppose without loss of generality that $f \geq 0$. By (1.9) and Lemma 8 we have

$$
\sum_{n \in S(x, y)} f(n)=\Psi(x, y) H_{f}^{*}(x, y)+O\left(R_{5}\right),
$$

where

$$
R_{5}=\Psi(x, y) \sum_{p^{k} \leq x, p \leq y} \frac{f\left(p^{k}\right)}{p^{k} \varrho^{*}(u)} \varrho^{*}\left(u-\frac{\log p^{k}}{\log y}\right)\left(u-\frac{\log p^{k}}{\log y}+1\right)^{-1} .
$$

Applying the Cauchy-Schwarz inequality and then using Lemmas 11 and 13 and (1.13) yields

$$
\begin{aligned}
R_{5} & \ll \Psi(x, y) \sqrt{\Theta_{f}^{*}(x, y)}\left\{u^{-1} H^{*}(x, y)^{1 / 2}+\left(\Sigma_{B}\right)^{1 / 2}\right\} \\
& \ll \Psi(x, y) \sqrt{u^{-1} \Theta_{f}^{*}(x, y)} .
\end{aligned}
$$

Similarly,

$$
H_{f}^{*}(x, y) \ll \sqrt{u \Theta_{f}^{*}(x, y)} .
$$

By (5.1)-(5.3) we further have

$$
\begin{aligned}
W^{*} & :=\sum_{n \in S(x, y)}\left\{f(n)-H_{f}^{*}(x, y)\right\}^{2} \\
& =\sum_{n \in S(x, y)} f^{2}(n)-\Psi(x, y) H_{f}^{*}(x, y)^{2}+O\left(\Psi(x, y) \Theta_{f}^{*}(x, y)\right) .
\end{aligned}
$$


For fixed $y$, put $\log p^{k} / \log y=t, \log q^{l} / \log y=s, \alpha\left(x / p^{k}, y\right)=\alpha_{u-t}$, $\alpha\left(x / q^{l}, y\right)=\alpha_{u-s}, \alpha\left(x /\left(p^{k} q^{l}\right), y\right)=\alpha_{u-t-s}$. By (1.9) and Lemma 8 we have

$$
\sum_{n \in S(x, y)} f^{2}(n) \leq W_{1}^{*}+W_{2}^{*}
$$

where

$$
\begin{aligned}
W_{1}^{*}= & \Psi(x, y) \sum_{\substack{p^{k} q^{l} \leq x \\
p \leq y, q \leq y}} \frac{f\left(p^{k}\right) f\left(q^{l}\right)}{p^{k} q^{l} \varrho^{*}(u)} \varrho^{*}(u-t-s)\left(1-p^{-\alpha_{u-t-s}}\right) \\
& \times\left(1-q^{-\alpha_{u-t-s}}\right)\left(1+O\left((u-t-s+1)^{-1}\right)\right)
\end{aligned}
$$

and

$$
W_{2}^{*}=\sum_{p^{k} \leq x, p \leq y} f^{2}\left(p^{k}\right)\left\{\Psi\left(\frac{x}{p^{k}}, y\right)-\Psi\left(\frac{x}{p^{k+1}}, y\right)\right\}
$$

Since

$$
\begin{aligned}
& \left(1-p^{-\alpha_{u-t-s}}\right)\left(1-q^{-\alpha_{u-t-s}}\right) \\
& =\left(1-p^{-\alpha_{u-t}}\right)\left(1-q^{-\alpha_{u-s}}\right)+O\left(p^{-\alpha_{u-t}}-p^{-\alpha_{u-t-s}}\right)+O\left(q^{-\alpha_{u-s}}-q^{-\alpha_{u-t-s}}\right),
\end{aligned}
$$

by Lemma 10 we have

$$
W_{1}^{*} \leq \Psi(x, y) H_{f}^{*}(x, y)^{2}+O\left(R_{6}\right)+O\left(R_{7}\right)+O\left(R_{8}\right),
$$

where

$$
\begin{aligned}
& R_{6}=\Psi(x, y) \sum_{\substack{p^{k} q^{l} \leq x \\
p \leq y, q \leq y}} \frac{f\left(p^{k}\right) f\left(q^{l}\right)}{p^{k} q^{l} \varrho^{*}(u)^{2}} \varrho^{*}(u-t) \varrho^{*}(u-s)(u-t-s+1)^{-1}, \\
& R_{7}=\Psi(x, y) \sum_{\substack{p^{k} q^{l} \leq x \\
p \leq y, q \leq y}} \frac{f\left(p^{k}\right) f\left(q^{l}\right)}{p^{k} q^{l} \varrho^{*}(u)^{2}} \varrho^{*}(u-t) \varrho^{*}(u-s)\left(p^{-\alpha_{u-t}}-p^{-\alpha_{u-t-s}}\right), \\
& R_{8}=\Psi(x, y) \sum_{\substack{p^{k} q^{l} \leq x \\
p \leq y, q \leq y}} \frac{f\left(p^{k}\right) f\left(q^{l}\right)}{p^{k} q^{l} \varrho^{*}(u)^{2}} \varrho^{*}(u-t) \varrho^{*}(u-s)\left(\frac{s}{u}\right) .
\end{aligned}
$$

Applying the Cauchy-Schwarz inequality, and then using Lemmas 11 and 13 and (1.13) yields

$$
\begin{aligned}
R_{6} & \ll \Psi(x, y) \Theta_{f}^{*}(x, y)\left(H_{f}^{*}(x, y)^{2} u^{-2}+H_{f}^{*}(x, y)\left(\Sigma_{B}\right)\right)^{1 / 2} \\
& \ll \Psi(x, y) \Theta_{f}^{*}(x, y) .
\end{aligned}
$$

It is easy to prove that

$$
p^{-\alpha_{u-t}}-p^{-\alpha_{u-t-s}} \ll p^{-\alpha_{u-t}}\left(\frac{s}{u}\right) \ll \frac{s}{u} .
$$


Hence $R_{7} \ll R_{8}$. Applying the Cauchy-Schwarz inequality and then using Lemmas 11 and 12 we have

$$
R_{8} \ll \Psi(x, y) \Theta_{f}^{*}(x, y)\left(u^{-2} H^{*}(x, y)\left(\Sigma_{A}\right)\right)^{1 / 2} \ll \Psi(x, y) \Theta_{f}^{*}(x, y) .
$$

Obviously,

$$
W_{2}^{*} \ll \Psi(x, y) \Theta_{f}^{*}(x, y) .
$$

Collecting (5.5)-(5.9) yields

$$
\sum_{n \in S(x, y)} f^{2}(n) \leq \Psi(x, y) H_{f}^{*}(x, y)^{2}+O\left(\Psi(x, y) \Theta_{f}^{*}(x, y)\right) .
$$

From this and (5.4), the proof of Theorem 3 is now complete.

Proof of Corollary 2. We may suppose without loss of generality that $f \geq 0$. We have

$$
H_{f}^{*}(x, y)=\bar{H}_{f}^{*}(x, y)-\Delta^{*}(x, y),
$$

where

$$
\Delta^{*}(x, y)=\sum_{p^{k} \leq x, p \leq y} \frac{f\left(p^{k}\right)}{p^{k} \varrho^{*}(u)} \varrho^{*}\left(u-\frac{\log p^{k}}{\log y}\right) p^{-\alpha\left(x / p^{k}, y\right)} .
$$

By (5.1), (5.2) and (5.11) we have

$$
\begin{aligned}
& \sum_{n \in S(x, y)}\left\{f(n)-\bar{H}_{f}^{*}(x, y)\right\}^{2} \\
& =\sum_{n \in S(x, y)} f^{2}(n)-\Psi(x, y) \bar{H}_{f}^{*}(x, y)-2 \Psi(x, y) \bar{H}_{f}^{*}(x, y) \Delta^{*}(x, y) \\
& \quad+O\left(\Psi(x, y) \Theta_{f}^{*}(x, y)\right) .
\end{aligned}
$$

Now (5.10) and (5.11) imply

$$
\begin{aligned}
\sum_{n \in S(x, y)} f^{2}(n) \leq & \Psi(x, y) \bar{H}_{f}^{*}(x, y)^{2}-2 \Psi(x, y) \bar{H}_{f}^{*}(x, y) \Delta^{*}(x, y) \\
& +\Psi(x, y) \Delta^{*}(x, y)^{2}+O\left(\Psi(x, y) \Theta_{f}^{*}(x, y)\right)
\end{aligned}
$$

Applying the Cauchy-Schwarz inequality and Lemma 9 we obtain

$$
\begin{aligned}
\Delta^{*}(x, y)^{2} & \leq \Theta_{f}^{*}(x, y) \sum_{p^{k} \leq x, p \leq y} \frac{1}{p^{k} \varrho^{*}(u)} \varrho^{*}(u-t) p^{-2 \alpha_{u-t}} \\
& \ll \Theta_{f}^{*}(x, y) \sum_{p^{k} \leq x, p \leq y} \frac{1}{p^{k+2}} p^{(k+2)\left(1-\alpha_{u}\right)} .
\end{aligned}
$$

(7.8) of [6] implies the last sum is $\ll 1$, for $x, y$ satisfying (4.18) and $x_{0}$ sufficiently large, hence

$$
\Delta^{*}(x, y) \ll \Theta_{f}^{*}(x, y) .
$$

Combining (5.12)-(5.14) completes the proof of Corollary 2. 
6. Examples. Let

$$
\beta(n)=\sum_{p \mid n} p, \quad B(n)=\sum_{p^{\alpha} \| n} \alpha p, \quad B_{1}(n)=\sum_{p^{\alpha} \| n} p^{\alpha},
$$

where $p^{\alpha} \| n$ means that $p^{\alpha}$ divides $n$ ( $p$ prime), but $p^{\alpha+1}$ does not.

The functions $\beta(n), B(n)$ and $B_{1}(n)$ are additive, and they are in a sense "large" (the average order is $\pi^{2} n /(6 \log n)$, see [2]).

Proposition 1. For $x$, y satisfying (1.6) we have uniformly

$$
\eta_{\beta}(x, y)=\operatorname{li}\left(y e^{\xi(u)}\right)\left(1+O\left(\frac{1}{u}\right)\right)
$$

and

$$
\theta_{\beta}(x, y)=\operatorname{li}\left(y^{2} e^{\xi(u)}\right)\left(1+O\left(\frac{1}{u}\right)\right),
$$

where $\eta_{f}(x, y)$ and $\theta_{f}(x, y)$ are defined as in [1].

Proposition 2. For $x$, y satisfying (1.6) we have uniformly

$$
\bar{H}_{B}(x, y)=\operatorname{li}\left(y e^{\xi(u)}\right)\left(1+O\left(\frac{1}{u}\right)\right)
$$

and

$$
\Theta_{B}(x, y)=\operatorname{li}\left(y^{2} e^{\xi(u)}\right)\left(1+O\left(\frac{1}{u}\right)\right)
$$

where $\bar{H}_{f}(x, y)$ and $\Theta_{f}(x, y)$ are defined as in Section 1.

Proposition 3. For $x$, y satisfying (1.6) we have uniformly

$$
\begin{aligned}
\bar{H}_{B_{1}}(x, y)= & \frac{c(u)}{\varrho(u)} \frac{y}{\log y}\left(1+O\left(\frac{u}{\log y}\right)\right), \\
\Theta_{B_{1}}(x, y)= & \frac{1}{\varrho(u)}\left\{\frac{y^{[u]+1}}{([u]+1) \log y}\right. \\
& \left.+\frac{([u]+1) y^{u+u /([u]+1)}}{([u]+2) u \log y}\right\}\left(1+O\left(\frac{1}{\log y}\right)\right),
\end{aligned}
$$

where $[x]$ denotes the greatest integer not exceeding the real number $x$, and $c(u)=\sum_{1 \leq k \leq[u]} \varrho(u-k)$, and $1 \leq c(u)<3$.

Remarks. (i) It is easily seen that for $1 \leq u \leq(\log y)^{1-\varepsilon}$,

$$
\begin{aligned}
& \eta_{\beta}\left(y^{u}, y\right) \sim \bar{H}_{B}\left(y^{u}, y\right) \quad \text { as } y \rightarrow \infty, \\
& \theta_{\beta}\left(y^{u}, y\right) \sim \Theta_{B}\left(y^{u}, y\right) \quad \text { as } y \rightarrow \infty,
\end{aligned}
$$

and that for $2 \leq u \leq(\log y)^{1-\varepsilon}$ and $y \geq y_{0}$ ( $y_{0}$ is a sufficiently large constant),

$$
\bar{H}_{B_{1}}\left(y^{u}, y\right)>c_{2} \bar{H}_{B}\left(y^{u}, y\right) \quad(c>1)
$$


and

$$
\Theta_{B_{1}}\left(y^{u}, y\right)>y^{1 / 2} \Theta_{B}\left(y^{u}, y\right) .
$$

From this we see that the generalization obtained in Theorem 1 is in a sense non-trivial.

(ii) We emphasize that in the classical case $y=x$,

$$
\eta_{\beta}(x, x) \sim \bar{H}_{B}(x, x) \sim \bar{H}_{B_{1}}(x, x) \quad \text { as } x \rightarrow \infty
$$

and

$$
\theta_{\beta}(x, x) \sim \Theta_{B}(x, x) \sim \Theta_{B_{1}}(x, x) \quad \text { as } x \rightarrow \infty .
$$

We will only prove Proposition 3 ; the proofs of Propositions 1 and 2 are similar but simpler.

Proof of Proposition 3. By the definition of $\bar{H}_{f}(x, y)$, we have

$$
\begin{aligned}
\bar{H}_{B_{1}}(x, y)= & \frac{1}{\varrho(u)}\left\{\sum_{1 \leq k \leq u} \sum_{p \leq y} \varrho\left(u-\frac{\log p^{k}}{\log y}\right)\right. \\
& \left.+\sum_{[u]+1 \leq k \leq \log x / \log 2} \sum_{p \leq x^{1 / k}} \varrho\left(u-\frac{\log p^{k}}{\log y}\right)\right\} \\
= & \frac{1}{\varrho(u)}\left(\Sigma_{1}+\Sigma_{2}\right), \quad \text { say . }
\end{aligned}
$$

Partial summation and the prime number theorem yield

$$
\begin{aligned}
\sum_{p \leq y} \varrho\left(u-\frac{k \log p}{\log y}\right)= & \int_{2}^{y} \varrho\left(u-\frac{k \log z}{\log y}\right) \frac{d z}{\log z}\left(1+O\left(\frac{1}{\log y}\right)\right) \\
& +O\left(\int_{2}^{y}\left|\varrho^{\prime}\left(u-\frac{k \log z}{\log y}\right)\right| \frac{k}{\log y} \frac{d z}{z}\right) \\
= & I_{1}+O\left(I_{2}\right), \quad \text { say. }
\end{aligned}
$$

In the first integral we make the change of variable $w=\log z / \log y$ and obtain

$$
I_{1}=\int_{\delta}^{1} \varrho(u-k w) y^{w} \frac{d w}{w}\left(1+O\left(\frac{1}{\log y}\right)\right),
$$

where $\delta=\log 2 / \log y$. Now let us apply integration by parts in such a manner that $y^{w}$ is chosen as the factor to be integrated, which results in the appearance of a factor $1 / \log y$. By routine calculations we obtain

$$
I_{1}=\varrho(u-k) \frac{y}{\log y}\left(1+O\left(\frac{1}{\log y}\right)\right)+O\left(\frac{k \varrho(u-k-1)}{u-k+1} \frac{y}{\log ^{2} y}\right),
$$


and similarly,

$$
I_{2} \ll \frac{y}{\log ^{2} y} \frac{k}{u-k+1} \varrho(u-k-1) \quad(k \leq u-1) .
$$

This implies that

$$
\Sigma_{1}=\frac{y}{\log y} \sum_{1 \leq k \leq u} \varrho(u-k)\left(1+O\left(\frac{u}{\log y}\right)\right) .
$$

To estimate $\Sigma_{2}$, we apply again partial summation and the prime number theorem to obtain

$$
\sum_{p \leq x^{1 / k}} \varrho\left(u-\frac{k \log p}{\log y}\right) \ll \frac{k x^{1 / k}}{\log x}+\frac{k^{2} x^{1 / k}}{\log x \log y},
$$

so that

$$
\Sigma_{2} \ll \frac{u y^{u /([u]+1)}}{\log ^{2} y} .
$$

This provides the required estimate. Also

$$
\begin{aligned}
c(u) & =\sum_{1 \leq k \leq[u]} \varrho(u-k) \\
& \leq 1+\varrho(1)+\varrho(2)+\ldots+\varrho([u]-1) \\
& \leq 1+\int_{0}^{\infty} \varrho(u) d u=1+e^{\gamma}<3 .
\end{aligned}
$$

It remains to prove the estimate (6.2). We write

$$
\begin{aligned}
\Theta_{B_{1}}(x, y)= & \frac{1}{\varrho(u)}\left\{\sum_{1 \leq k \leq u} \sum_{p \leq y} p^{k} \varrho\left(u-\frac{\log p^{k}}{\log y}\right)\right. \\
& \left.+\sum_{[u]+1 \leq k \leq \log x / \log 2} \sum_{p \leq x^{1 / k}} p^{k} \varrho\left(u-\frac{\log p^{k}}{\log y}\right)\right\} \\
= & \frac{1}{\varrho(u)}\left(\Sigma_{3}+\Sigma_{4}\right), \quad \text { say . }
\end{aligned}
$$

Partial summation and the prime number theorem yield

$$
\begin{aligned}
\sum_{p \leq y} p^{k} \varrho(u & \left.-\frac{k \log p}{\log y}\right) \\
& =\int_{\log 2}^{\log y} e^{(k+1) w} \varrho\left(u-\frac{k w}{\log y}\right) \frac{d w}{w}\left(1+O\left(\frac{1}{\log ^{2} y}\right)\right) .
\end{aligned}
$$


Using integration by parts we deduce that the right-hand side of (6.4) is equal to

$$
\begin{aligned}
\frac{y^{k+1}}{(k+1) \log y} \varrho(u-k)\left(1+O\left(\frac{1}{\log ^{2} y}\right)\right) \\
+O\left(\int_{\log 2}^{\log y} \frac{e^{(k+1) w}}{k+1} \varrho\left(u-\frac{k w}{\log y}\right) \frac{d w}{w^{2}}\right) \\
+O\left(\int_{\log 2}^{\log y} \frac{e^{(k+1) w}}{k+1}\left|\varrho^{\prime}\left(u-\frac{k w}{\log y}\right)\right| \frac{k}{\log y} \frac{d w}{w}\right) .
\end{aligned}
$$

The first error term is of order

$$
\ll \frac{y^{k+1}}{(k \log y)^{2}} \varrho(u-k) .
$$

By the definition of $\varrho(u)$, the second error term can be bounded, for $k \leq u$, by

$$
\ll \frac{k}{\log y} \frac{\varrho(u-k)}{u-k+1} \int_{\log 2}^{\log y} \frac{e^{(k+1) w}}{k+1} \frac{d w}{w} \ll \frac{y^{k+1}}{k \log ^{2} y} .
$$

Thus, we obtain

$$
\Sigma_{3}=\frac{y^{[u]+1}}{([u]+1) \log y}\left(1+O\left(\frac{1}{\log y}\right)\right) .
$$

Moreover, we may deduce in the same way as before

$$
\begin{aligned}
\Sigma_{4} & =\sum_{[u]+1 \leq k \leq \log x / \log 2} \frac{k x^{(k+1) / k}}{(k+1) \log x}\left(1+O\left(\frac{1}{\log x}\right)\right) \\
& =\frac{([u]+1) x^{([u]+2) /([u]+1)}}{([u]+2) \log x}\left(1+O\left(\frac{1}{\log x}\right)\right) .
\end{aligned}
$$

Thus the proof of (6.2) is complete.

\section{References}

[1] K. Alladi, The Turán-Kubilius inequality for integers without large prime factors, J. Reine Angew. Math. 335 (1982), 180-196.

[2] K. Alladi and P. Erdős, On an additive arithmetic function, Pacific J. Math. 71 (1977), 275-294.

[3] N. G. de Bruijn, On the number of positive integers $\leq x$ and free of prime factors $>y$, Indag. Math. 13 (1951), 50-60.

[4] P. D. T. A. Elliott, Probabilistic Number Theory, Vol. 1, Springer, Berlin, 1980.

[5] A. Hildebrand, On the number of positive integers $\leq x$ and free of prime factors $>y$, J. Number Theory 22 (1986), 289-307. 
[6] A. Hild ebrand and G. Tenenbaum, On integers free of large prime factors, Trans. Amer. Math. Soc. 296 (1986), 265-290.

[7] T. Z. Xuan, The average order of $d_{k}(n)$ over integers free of large prime factors, Acta Arith. 55 (1990), 249-260.

[8] - The Turán-Kubilius inequality for integers free of large prime factors, J. Number Theory 43 (1993), 82-87.

DEPARTMENT OF MATHEMATICS

BEIJING NORMAL UNIVERSITY

BEIJING 100875

PEOPLE'S REPUBLIC OF CHINA 